\title{
Adubação nitrogenada para a cana-de-açúcar colhida crua, em rotação com leguminosas, cultivada em solo de tabuleiro costeiro
}

\section{Sugarcane response to nitrogen application in a no burn harvesting system, in rotation with legumes, for a coastal tableland soil}

\author{
Paulo de Albuquerque Silva ${ }^{1}$; Walane Maria Pereira de Mello Ivo ${ }^{2}$; Antonio Dias Santiago 3
}

${ }^{1}$ Doutor em Fitotecnia e Pesquisador da Embrapa Tabuleiros Costeiros, Unidade de Execução de Pesquisa de Rio Largo, Rio Largo, (82) 3261-1322, paulo.dealbuquerque@embrapa.br; ${ }^{2}$ Doutora em Aplicação de Radioisótopos na Agricultura e Meio Ambiente e Pesquisadora da Embrapa Tabuleiros Costeiros, Unidade de Execução de Pesquisa de Rio Largo, Rio Largo, walane.ivo@embrapa.br; ${ }^{3}$ Doutor em Fitotecnia e Pesquisador da Embrapa Tabuleiros Costeiros-Unidade de Execução de Pesquisa de Rio Largo, Rio Largo, antonio.santiago@embrapa.br

\section{A R T I G O}

Recebido: $15 / 12 / 2019$

Aprovado: 20/12/2019

\section{Palavras-chave:}

Crotalaria spectabilis.

Nitrogênio

Saccharum officinarum

Key words:

Crotalaria spectabilis

Nitrogen

Saccharum officinarum

\section{R E S U M O}

A incorporação de material de elevada relação Carbono/Nitrogênio originário da colheita sem despalha a fogo e os novos cultivares de cana-de-açúcar podem determinar recomendações mais elevadas de adubação nitrogenada para a cultura. Como a adubação nitrogenada tem importante papel nas emissões de gases de efeito estufa, uma forma de diminuir essas emissões pode ser a substituição parcial dos fertilizantes por leguminosas, em rotação com a cana. O objetivo deste trabalho é estabelecer a recomendação de adubação nitrogenada para a cana-de-açúcar colhida crua e cultivada em rotação com leguminosas. Os tratamentos avaliados são: variedades de cana (RB 92579 e RB 867515), rotação com Crotalaria spectabilis, e doses de N (0 e $60 \mathrm{~kg} \mathrm{ha}^{-1}$ para cana planta e, nas duas socarias, $0,60,120$ e $180 \mathrm{~kg} \mathrm{de} \mathrm{N} \mathrm{ha}^{-1}$ ). A resposta da cana planta à rotação com crotalaria e à aplicação de $60 \mathrm{~kg}$ de $\mathrm{N} \mathrm{ha}^{-1}$ é expressiva, com a indicação de que a cana só responde ao $\mathrm{N}$ fertilizante na ausência da leguminosa. $\mathrm{O}$ nitrogênio da fertilização nitrogenada resulta em resposta quadrática na produtividade de colmos da socaria da cana, estabelecendo recomendação de doses de 124 a $146 \mathrm{~kg} \mathrm{ha}^{-1}$ de $\mathrm{N}$, para materiais de alto rendimento.

\section{A B S T R A C T}

Incorporation of high carbon/nitrogen material, from sugarcane harvested without burn, and new sugarcane cultivars, recomended in the last years, may lead to higher recommendations for nitrogen fertilization. As nitrogen fertilization plays an important role in greenhouse gas emissions, one way to reduce these emissions can be the partial replacement of fertilizers with legumes rotating, with sugarcane. The objective of this work is to establish the recommendation of nitrogen fertilization for sugarcane harvested without burn, and cultivated in rotation with Crotalaria spectabilis. The evaluated treatments are: sugarcane varieties (RB 92579 and RB $867515)$, rotation with Crotalaria spectabilis, and $\mathrm{N}$ rates ( 0 and $60 \mathrm{~kg} \mathrm{ha}^{-1}$ for first cicle and, in two subsequent rattons, $0,60,120$ and $180 \mathrm{~kg} \mathrm{~N} \mathrm{ha}^{-1}$ ). The response of sugarcane to Crotalaria rotation and application of $60 \mathrm{~kg}$ of $\mathrm{N} \mathrm{ha}^{-1}$ is significant, indicating that sugarcane responds to $\mathrm{N}$ fertilizer only in the absence of legume. Nitrogen from fertilization results in quadratic response of sugarcane ratoons yield, setting recommended doses of 124 to $146 \mathrm{~kg} \mathrm{ha}^{-1}$ of $\mathrm{N}$ for high yield materials.

\section{INTRODUÇÃO}

A colheita de cana sem despalha a fogo tem se intensificado no Brasil, em função de legislações e da possibilidade de uso industrial da palhada da cana. Neste sistema de produção, com a permanência dos resíduos na superfície do solo, poderá ocorrer, no curto e médio prazos, imobilização temporária do nitrogênio, como consequiência da incorporação de material de elevada relação Carbono/Nitrogênio (C:N = 80 a 100) (SILVA, 2013). Tal fato pode fazer com que a cana planta responda ao $\mathrm{N}$, ainda mais em colheita crua e sem revolvimento. Além disto, os cultivares de cana recomendados nos últimos anos tem se mostrado mais produtivos e, desta forma, necessitam de reposição de maiores quantidades de nutrientes no sistema de produção da cultura.

\section{Revista Verde}

ISSN 1981-8203

Pombal, Paraíba, Brasil v. 14, n.5, Edição Especial, p.635-640, 2019

doi: $10.18378 /$ rvads.v14i5.7612

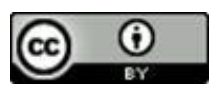


Os estudos de acúmulo de nutrientes pela cana-de-açúcar mostram que, para uma produtividade de $100 \mathrm{t}$ de colmos, a planta absorve $\mathrm{N}$ do solo, do fertilizante, da atmosfera, em torno de 200 a $300 \mathrm{~kg} \mathrm{ha}^{-1}$ de $\mathrm{N}$; e que, do $\mathrm{N}$ extraído pela cana, $50 \%$ é exportado pelos colmos, levando a uma quantidade de $\mathrm{N}$ acumulado de $1,4 \mathrm{~kg}$ de $\mathrm{N}$ por tonelada de colmo. Isso mostra que as quantidades exportadas chegam a ser maiores que as doses aplicadas. Por outro lado, no longo prazo (30 a 40 anos), existe a previsão de redução da dose de fertilizantes nitrogenados a serem recomendados para a cana-de-açúcar colhida crua (GAVA et al., 2003).

Todos estes fatores podem levar a recomendações de doses de $\mathrm{N}$, e de outros nutrientes, diferentes daquelas recomendadas anteriormente, quando no sistema de produção da cultura utilizava-se o fogo, antes da colheita. Desta forma, é preciso ajustar as doses de $\mathrm{N}$ a serem recomendadas nas adubações por fertilizantes e, também, pelo uso de adubos verdes. Com relação a estes últimos, como na região Nordeste a cana-de-açúcar é plantada principalmente nos Tabuleiros Costeiros, em solos com limitações química e física, uma das alternativas utilizadas para aumentar a profundidade efetiva dos solos dos Tabuleiros Costeiros com horizontes coesos vem sendo o uso de práticas mecânicas (subsolagem), práticas biológicas (leguminosas e/ou adubação orgânica) ou a associação das duas práticas.

A cultura da cana-de-açúcar tem uma importante representatividade no cenário da agricultura da região, além de apresentar balanço de emissões de gases de efeito estufa promissor, principalmente por emissões evitadas. Estas são as emissões que ocorreriam se, na ausência do etanol e do bagaço excedente, a demanda de combustíveis fosse satisfeita por gasolina e óleo combustível. Porém, esses valores podem ainda ser melhorados se o sistema de produção da cana-de-açúcar for trabalhado no sentido de reduzir as emissões reais. Devem ser reduzidas as emissões relacionadas às fontes de emissão agrícola como àquelas provenientes de uso de fertilizantes nitrogenados, uso de diesel em operações de máquinas, queima da cana, resíduos (vinhaça, torta de filtro) e calagem.

Como a produção de $\mathrm{N}_{2} \mathrm{O}$ pela nitrificação ou desnitrificação depende do $\mathrm{N}$ disponível no solo, a aplicação de fertilizante e resíduos é um controlador importante das emissões deste gás. Porém, de acordo com Bayer et al. (2011), uma forma de reduzir emissões de $\mathrm{N}_{2} \mathrm{O}$ para a atmosfera pode ser a substituição parcial da adubação nitrogenada pela utilização de leguminosas, uma vez que a liberação mais lenta do $\mathrm{N}$ contido no resíduo vegetal, comparativamente com o $\mathrm{N}$ inorgânico, provavelmente permite que o $\mathrm{N}$ mineral seja gradativamente absorvido pelas plantas em desenvolvimento, reduzindo, assim, o potencial de emissão de $\mathrm{N}_{2} \mathrm{O}$. O que poderá ocorrer em áreas de cana com rotação com leguminosas.

O objetivo deste trabalho é estabelecer a recomendação de adubação nitrogenada para a cana-de-açúcar colhida crua e cultivada em solo de tabuleiro, em rotação com leguminosas.

\section{MATERIAL E MÉTODOS}

$\mathrm{O}$ trabalho foi conduzido em área da Usina Coruripe, no município de Coruripe, Alagoas ( $\left.10^{\circ} 08^{\prime} \mathrm{S} ; 36^{\circ} 18^{\prime} \mathrm{O}\right)$. O solo da área foi classificado como um Argissolo Amarelo. A precipitação anual média é de $1500 \mathrm{~mm}$, concentrada no período de maio a setembro, e temperatura do ar em torno de 28 ${ }^{\circ} \mathrm{C}$. A Crotalaria spectabilis foi estabelecida em área de canade-açúcar, a ser colhida sem despalha a fogo, em final de maio e, em início de agosto, a área foi sulcada para o plantio da cana.

$\mathrm{O}$ delineamento experimental foi em blocos arranjados em faixas, com 4 repetições. Nas parcelas principais ficaram as duas variedades de cana, RB 92579 e RB 867515, nas subparcelas os tratamentos com e sem leguminosas, e nas sub-sub parcelas as diferentes doses de $\mathrm{N}\left(0\right.$ e $60 \mathrm{~kg} \mathrm{ha}^{-1}$ no primeiro ano). Para a cana planta, cada uma destas sub-sub parcelas foi composta por 40 linhas de $10 \mathrm{~m}$ e, na socaria, estas sub-sub parcelas foram formadas por 9 linhas de $10 \mathrm{~m}$, nas quais foram aplicadas as doses de $0,60,120$ e $180 \mathrm{~kg}$ de $\mathrm{N} \mathrm{ha}^{-1}$, nas duas socarias seguintes. A massa seca da leguminosa - crotalária espectábilis - foi determinada antes do plantio da cana, coletando-se 8 repetições de $1 \mathrm{~m}^{2}$, por tratamento. O material coletado foi limpo e, em seguida, seco em estufa à temperatura de $65{ }^{\circ} \mathrm{C}$, até peso constante, pesado e enviado para análise química para determinação dos teores de $\mathrm{N}, \mathrm{P}, \mathrm{K}, \mathrm{Ca}, \mathrm{Mg}, \mathrm{S}$ e carbono (SILVA, 1999).

Nas parcelas com rotação, a leguminosa não foi incorporada ao solo, fazendo-se apenas os sulcos de plantio da cana sobre a massa verde da crotalária. Nesta ocasião foram aplicados $144 \mathrm{~kg} \mathrm{~K} \mathrm{ha}^{-1}$ e $100 \mathrm{~kg} \mathrm{P}_{2} \mathrm{O}_{5} \mathrm{ha}^{-1}, 30 \mathrm{~kg} \mathrm{ha}^{-1} \mathrm{de}$ fórmula de micronutrientes utilizada pela usina e $400 \mathrm{~g} \mathrm{ha}^{-1} \mathrm{de}$ molibidato de sódio. A adubação nitrogenada com ureia $(0 \mathrm{~kg}$ de $\mathrm{N} \mathrm{ha}^{-1}$ e $60 \mathrm{~kg}$ de $\mathrm{N} \mathrm{ha}^{-1}$ ) ocorreu aos 46 dias após o plantio da cana planta, com aplicação de irrigação logo em seguida. Dois meses após esta adubação, foi realizada a coleta de folhas para determinação de nutrientes, sendo coletadas dez folhas por parcela. A folha coletada foi a primeira folha com a aurícula totalmente aberta (folha +1 ). Nestas, foram determinados os seguintes nutrientes: $\mathrm{N}, \mathrm{P}, \mathrm{K}, \mathrm{Ca}, \mathrm{Mg}, \mathrm{S}$ e, também o carbono, de acordo com Silva (1999). A colheita ocorreu no mês de outubro, sendo colhidas quatro linhas de cada parcela. O material colhido foi pesado, para determinação da produtividade, e em seguida enviado para análises tecnológicas.

Para o ciclo das socarias também foi utilizada a ureia como fonte de $\mathrm{N}$, sendo a mesma aplicada sobre a palhada da cana planta, 50 dias após a colheita da cana, seguida imediatamente de irrigação. Para a primeira socaria também foram realizadas análises de teores de nutrientes na folha. Dois meses após a adubação de cobertura, foi realizada a coleta da primeira folha com a aurícula totalmente aberta (folha +1 ), sendo coletadas dez folhas por parcela. Nestas, também foram determinados os seguintes nutrientes: $\mathrm{N}, \mathrm{P}, \mathrm{K}, \mathrm{Ca}, \mathrm{Mg}, \mathrm{S}$ e, também o carbono, de acordo com Silva (1999). As colheitas destes ciclos ocorreram na primeira quinzena de dezembro, sendo colhidas e pesadas as quatro fileiras centrais das parcelas, onde amostras de colmos foram encaminhadas para a execução de análises tecnológicas.

Para a cana planta os dados de nutrientes na folha e de produtividade foram analisados por meio de análises de variância e pela comparação de médias realizada pelo teste-t de Tukey a 5\% de probabilidade. Análises de regressão polinomial foram realizadas para os dados da primeira e segunda socaria, e, em seguida, foram determinadas as doses de máxima eficiência econômica para a adubação nitrogenada da cana-de-açúcar. 


\section{RESULTADOS E DISCUSSÃO}

\section{a) Cana Planta}

A produção de massa seca da parte aérea da C. spectabilis, por ocasião do florescimento, foi de $3,7 \mathrm{Mg} \mathrm{ha}^{-1}$. Além da parte aérea, mais $0,9 \mathrm{Mg} \mathrm{ha}^{-1}$ de material orgânico foram incorporados ao solo, via sistema radicular. A produtividade da massa seca da $C$. spectabilis foi inferior àquela encontrada por Barreto \& Fernandes (2001), na classificação de várias espécies de leguminosas quanto à sua capacidade de formação de biomassa da parte aérea, na região dos Tabuleiros Costeiros do Nordeste. A mesma foi classificada no grupo de produtividade intermediária $\left(6,4 \mathrm{Mg} \mathrm{ha}^{-1}\right.$ a $\left.6,1 \mathrm{Mg} \mathrm{ha}^{-1}\right)$; muito embora Barreto et al. (2014) descrevam valores de produtividade de até $9,9 \pm 1,9 \mathrm{Mg} \mathrm{ha}^{-1}$ para $C$. spectabilis na mesma região do presente estudo, em áreas da Usina Coruripe, com semeadura a lanço. Vale salientar que altas produções de biomassa por algumas leguminosas na região só são obtidas quando seu plantio é feito na época correta, no final de abril ou começo de maio, pois quando as mesmas são plantadas tardiamente, estas podem sofrer influência do fotoperíodo, reduzindo fortemente a produção de matéria seca da parte aérea.

Neste sentido, Silva et al (2016) estabeleceram como período mais adequado ao plantio de várias leguminosas na região dos tabuleiros costeiros a primeira e segunda quinzenas de maio. Após esta data as espécies estudadas apresentaram forte redução na produção de matéria seca. Mesmo com menor produção de biomassa, as quantidades de nutrientes adicionada e reciclada no sistema de produção da cana ainda foram consideráveis, com destaque para o nitrogênio (106 kg ha-1) e potássio $\left(67 \mathrm{~kg} \mathrm{ha}^{-1}\right)$. Este último é o elemento mais utilizado pela cana e, também, o exportado em maior quantidade, o que reforça a importância desta rotação com as leguminosas. Teores elevados de $\mathrm{Ca}$ no tecido da crotalária foram observados no presente estudo, estando em torno de $10 \mathrm{~g} \mathrm{~kg}^{-1}$, levando a reciclagem em superfície de $37 \mathrm{~kg} \mathrm{ha}^{-1}$ de Ca. Esta característica mostra-se muito interessante na rotação com a cana, quando uma variedade como a RB 92579 é plantada, pois esta se caracteriza como material de alto rendimento, sendo exigente em cálcio (OLIVEIRA et al, 2010).

Em resposta a esta rotação com a leguminosa e à aplicação do $\mathrm{N}$ fertilizante, houve aumento do teor de alguns nutrientes no tecido da cana-de-açúcar, cultivada em rotação. $\mathrm{O}$ teor de $\mathrm{N}$ respondeu tanto a incorporação da crotalária, como ao $\mathrm{N}$ fertilizante. Os outros nutrientes, como só estavam presentes na fonte orgânica (leguminosa), só responderam a esta. O que pode ser um indicativo de que o aumento no teor de $\mathrm{N}$ na planta, pela aplicação do fertilizante, não determinou aumento na absorção dos outros nutrientes. Franco et al. (2008), em estudo do acúmulo de $\mathrm{N}, \mathrm{P}$ e K, relacionados à adubação nitrogenada de plantio, não encontraram interferência da adubação nitrogenada nos teores de N, P e K, em todo o ciclo da cultura.

Tais diferenças relatadas para nutrientes nas folhas parecem ter influenciado a produtividade da cana-de-açúcar (Figura 1). Para esta variável, houve diferença entre as variedades de cana, com a RB 92579 apresentando-se mais produtiva que a RB 867515 , com os valores de produtividade de $167 \mathrm{Mg} \mathrm{ha}^{-1}$ e $125 \mathrm{Mg} \mathrm{ha}^{-1}$ de cana, respectivamente. Também ocorreu diferença significativa com relação ao uso da leguminosa, onde a presença desta promoveu aumento de produtividade da cana. A produtividade média das cultivares na área com crotalária foi de $163 \mathrm{Mg} \mathrm{ha}^{-1}$ de cana e, na área sem rotação com a leguminosa, foi de $132 \mathrm{Mg} \mathrm{ha}^{-1}$ de cana. Da mesma forma, para as doses de $\mathrm{N}$, a aplicação de $60 \mathrm{~kg} \mathrm{ha}^{-1}$ deste nutriente levou a uma produtividade mais elevada (156 $\left.\mathrm{Mg} \mathrm{ha}^{-1}\right)$, em relação às áreas sem aplicação do fertilizante (141 $\left.\mathrm{Mg} \mathrm{ha}{ }^{-1}\right)$. Além disso, houve interação entre o uso da leguminosa e o do $\mathrm{N}$ fertilizante, indicando que, a cana só responde ao $\mathrm{N}$ fertilizante na ausência da leguminosa (Figura 1). Desta forma, a redução na emissão de gases de efeito estufa pode ser alcançada no sistema de produção da cana-de-açúcar pela substituição da aplicação de fertilizantes nitrogenados por meio do uso de leguminosas em rotação com a cultura. Como exposto anteriormente, a liberação mais lenta do $\mathrm{N}$ contido no resíduo vegetal, comparativamente com o $\mathrm{N}$ inorgânico, provavelmente permite que o $\mathrm{N}$ mineral seja gradativamente absorvido pelas plantas em desenvolvimento, reduzindo, assim, o potencial de emissão de $\mathrm{N}_{2} \mathrm{O}$ (Bayer et al., 2011).

Figura 1. Produtividade da cana-de-açúcar $\left(\mathrm{Mg} \mathrm{ha}^{-1}\right)$, variedades (A) RB 92579 e (B) RB 867515, em resposta a aplicação de fertilizante nitrogenado $\left(0\right.$ e $60 \mathrm{~kg} \mathrm{ha}^{-1}$ de $\left.\mathrm{N}\right)$ e rotação com a leguminosa Crotalaria spectabilis. Coruripe, Alagoas (*Tukey a 5\% de probabilidade).
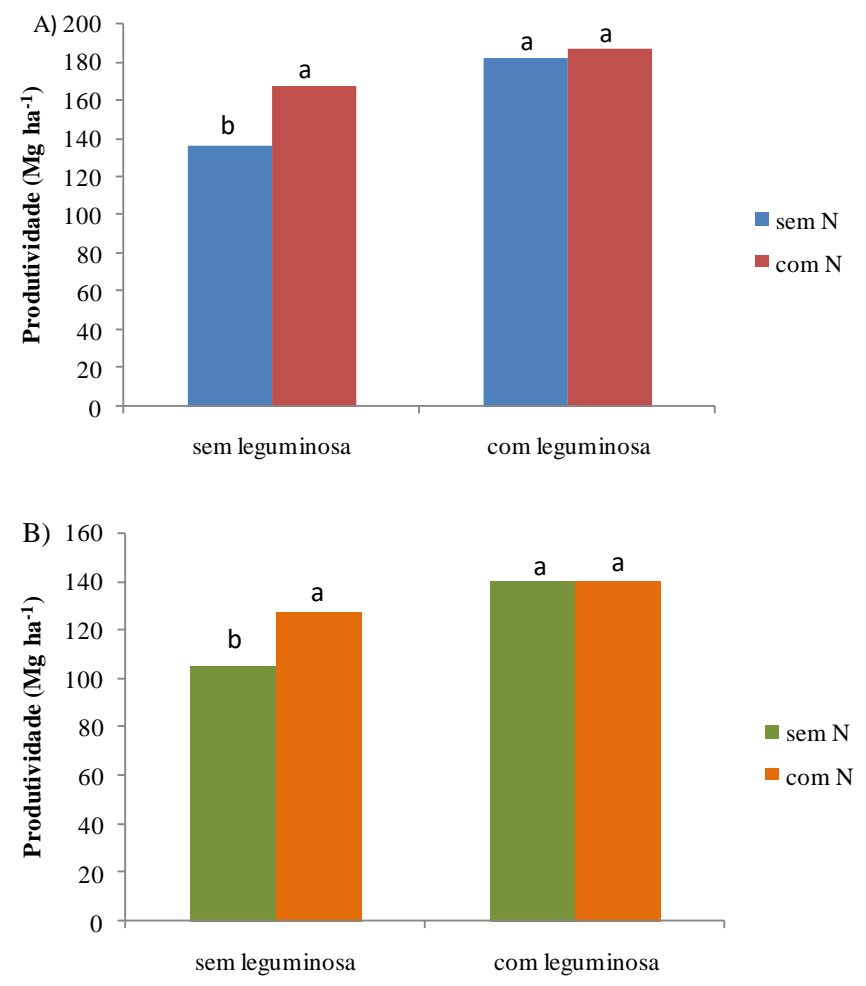

Bolonhezi et al. (2014) relatam aumentos de produtividade da cana, em função da rotação com leguminosas, em quantidades que variam de $10,9 \mathrm{Mg} \mathrm{ha}^{-1}$ a $42 \mathrm{Mg} \mathrm{ha}^{-1}$. No presente estudo, nas áreas sem aplicação de $\mathrm{N}$ fertilizante, esta diferença chegou a $44 \mathrm{Mg} \mathrm{ha}^{-1}$, representando um aumento de $27 \%$ na produtividade da cana, o que, segundos percentuais 
descritos pelos autores, não seria incomum em áreas que envolvem a prática da rotação com leguminosa.

\section{b) Primeira socaria}

As análises estatísticas indicaram que não houve efeito residual da leguminosa e do fertilizante, aplicados para a cana planta, sobre os teores de nutrientes no tecido da cana e na produtividade da primeira soca. Desta forma, as discussões se relacionam apenas a resposta da cana às diferentes doses de $\mathrm{N}$ $\left(0,60,120\right.$ e $180 \mathrm{~kg}$ de $\left.\mathrm{N} \mathrm{ha}^{-1}\right)$. Analisando-se a influência da fertilização nitrogenada nos teores de nutrientes no tecido da cana-de-açúcar, foi observado que o teor de nitrogênio na folha da cana aumentou com a elevação das doses de $\mathrm{N}$ aplicadas. Esses valores variaram de 13,1 a $19,5 \mathrm{~g} \mathrm{~kg}^{-1}$ para a RB 92579, e de 12,2 e 17,6 $\mathrm{g} \mathrm{kg}^{-1}$, para a RB 867515, nas doses de 0 e 180 $\mathrm{kg}$ de $\mathrm{N} \mathrm{ha}^{-1}$, respectivamente (Tabela 1). Este aumento dos teores foliares não se reflete para os outros nutrientes, ocorrendo apenas a tendência de aumento do teor de $\mathrm{P}$ e $\mathrm{Mg}$, até a dose de $120 \mathrm{~kg}$ de $\mathrm{N} \mathrm{ha}{ }^{-1}$, para a variedade $\mathrm{RB} 92579$. Também para esta variedade, houve uma redução clara do teor de potássio com o aumento da dose de $\mathrm{N}$ aplicada à cana-deaçúcar.

Tabela 1. Concentração de nutrientes na folha +1 (primeira folha com aurícula totalmente aberta) da primeira socaria de cana-de-açúcar, RB 92579 e RB 86 7515, cultivada em Argissolo Amarelo. Município de Coruripe, Alagoas.

\begin{tabular}{|c|c|c|c|c|c|c|}
\hline & $\mathrm{N}$ & $\mathrm{P}$ & $\mathrm{K}$ & $\mathrm{Ca}$ & $\mathrm{Mg}$ & $\mathrm{S}$ \\
\hline RB 92579 & \multicolumn{6}{|c|}{$\mathrm{g} \mathrm{kg}^{-1}$} \\
\hline \multicolumn{7}{|l|}{ Doses de N $\left(\mathrm{kg} \cdot \mathrm{ha}^{-1}\right)$} \\
\hline 0 & 13,08 & 1,78 & 7,21 & 2,33 & 0,65 & 0,79 \\
\hline 60 & 15,79 & 1,84 & 6,93 & 2,63 & 0,86 & 0,83 \\
\hline 120 & 18,37 & 2,12 & 6,54 & 2,79 & 1,03 & 0,95 \\
\hline 180 & 19,47 & 2,08 & 5,34 & 2,51 & 1,00 & 0,95 \\
\hline \multicolumn{7}{|l|}{ RB 867515} \\
\hline \multicolumn{7}{|l|}{ Doses de N $\left(\mathrm{kg} \cdot \mathrm{ha}^{-1}\right)$} \\
\hline 0 & 12,16 & 1,71 & 6,7 & 1,81 & 1,45 & 0,99 \\
\hline 60 & 14,81 & 1,98 & 7,2 & 2,26 & 1,43 & 1,16 \\
\hline 120 & 16,41 & 1,93 & 5,4 & 2,15 & 1,40 & 1,07 \\
\hline 180 & 17,61 & 1,93 & 6,9 & 2,06 & 1,74 & 1,15 \\
\hline
\end{tabular}

Os teores de nutrientes nas folhas da cana-de-açúcar encontraram-se abaixo dos limites determinados para esta cultura, quando cultivada em vários países do mundo (KORNDÖRFER et al., 2005), para o $\mathrm{N}$ e $\mathrm{Mg}$, sob a dose de 0 $\mathrm{kg}$ de $\mathrm{N} \mathrm{ha}^{-1}$; e, para o $\mathrm{K}$, nas maiores doses de $\mathrm{N}(120$ e $180 \mathrm{~kg}$ $\mathrm{N} \mathrm{ha}^{-1}$ ). Quando estes valores (Tabela 1) são comparados com os teores adequados, estabelecidos para os nutrientes no Estado de São Paulo (RAIJ et al., 1996; KORNDÖRFER et al., 2005), estes se apresentam abaixo dos limites inferiores em todas as doses, excetuando-se o $\mathrm{N}$, para $180 \mathrm{~kg}^{\mathrm{de}} \mathrm{N} \mathrm{ha}^{-1}$ (19,0 a 21,0 g $\mathrm{kg}^{-1}$ ), para a RB 92579; e, para o P, nas doses de 120 e $180 \mathrm{Kg}$ de $\mathrm{N}$ ha $^{-1}\left(2,0\right.$ a $\left.2,4 \mathrm{~g} \mathrm{~kg}^{-1}\right)$. Este fato parece não ser determinante para a região dos Tabuleiros Costeiros do Nordeste, uma vez que, apesar disto, as produtividades da cana foram altas (Figura 2). Neste sentido, Moura Filho et al. (2014) constataram valores ótimos para a cana-de-açúcar entre 12,520,8; 1,0-2,1; 8,9-14,7;1,7-4,0; 0,1-1,4; 0,7-1,8 g kg-1; para N, $\mathrm{P}, \mathrm{K}, \mathrm{Ca}, \mathrm{Mg}$ e $\mathrm{S}$, respectivamente, na região de Alagoas. Tais valores, considerados ótimos para a cultura, apresentam limites inferiores das faixas de variação mais baixos que os considerados para outras regiões do Brasil.

A resposta da cana soca a adubação nitrogenada foi observada no presente estudo. Polinômios de segundo grau foram ajustados para descrever as respostas da cana ao $\mathrm{N}(\mathrm{p}$ > 0,000) (Figura 2). Verificou-se que, como esperado, ao serem adicionadas quantidades crescentes de nitrogênio, o maior incremento na produção ocorreu com a primeira dose aplicada; e que, com aplicações sucessivas de quantidades iguais do nutriente (60 kg de $\mathrm{N} \mathrm{ha}^{-1}$ ), os incrementos de produção foram cada vez menores, como descrito por Raij (1981).
A resposta da primeira soca à aplicação de $\mathrm{N}$ pode ser explicada pelo maior teor foliar deste nutriente (Tabela 1). Observa-se que a maior produtividade de colmos esteve associada com o teor de $\mathrm{N}$ igual a $19,4 \mathrm{~g} \mathrm{~kg}^{-1}$, para a RB 92579, e igual a $17,6 \mathrm{~g} \mathrm{~kg}^{-1}$, para a RB 867515 . No entanto, os maiores ganhos da produtividade relacionaram-se com teores que variaram de 15,8 a 18,4 $\mathrm{g} \mathrm{kg}^{-1}$ (RB 92 579: 26 e $7 \mathrm{Mg}$ de colmos $\left.\mathrm{ha}^{-1}\right)$ e 14,8 e 16,4 $\mathrm{g} \mathrm{kg}^{-1}(\mathrm{RB} 86$ 7515: 8 e $7 \mathrm{Mg}$ de colmos ha $\left.{ }^{-1}\right)$. Desta forma, verifica-se uma maior eficiência da RB 92579, em relação à RB 867515, em resposta ao $\mathrm{N}$ aplicado, uma vez que o ganho inicial no peso de colmos foi bem maior para esta variedade.

Assim como para a cana planta, na socaria, a variedade RB 92579 apresentou maiores produtividades que a RB 867515 e, dentro de cada variedade, 0 ou $60 \mathrm{~kg}$ de $\mathrm{N} \mathrm{ha}^{-1}$ aplicados na cana planta não influenciou a resposta da socaria ao nutriente. Diferentemente, Vitti el al. (2007) encontraram um aumento linear na produtividade da segunda socaria da cana, em resposta a aplicação de doses crescentes de $\mathrm{N}$ até $175 \mathrm{~kg} \mathrm{ha}^{-1}$, com este efeito se estendendo à terceira socaria. Estes autores determinaram que em torno de $40 \%$ do amônio da fonte nitrato de amônio permanece no sistema solo-planta-palha como efeito residual para a safra seguinte, independente da dose de $\mathrm{N}$ aplicada; e que os estoques de $\mathrm{N}$ e $\mathrm{S}$ do sistema radicular no final da segunda safra se correlacionaram positivamente com a produtividade de colmos da cana na terceira soca. Desta forma, este nitrogênio residual da adubação na cana planta ou de socarias anteriores pode ter influência na produtividade do ciclo agrícola subsequente. 
Figura 2. Curva de resposta de cana-de-açúcar a aplicações de nitrogênio, para primeira socaria das variedades (A) RB 92579 e (B) RB 867515, cultivadas em Argissolo Amarelo, sem (0 N) e com $\left(60 \mathrm{~kg}\right.$ de $\left.\mathrm{N} \mathrm{ha}{ }^{-1} \mathrm{~N}\right)$ aplicação de nitrogênio na cana planta. Município de Coruripe, Alagoas.

(A)
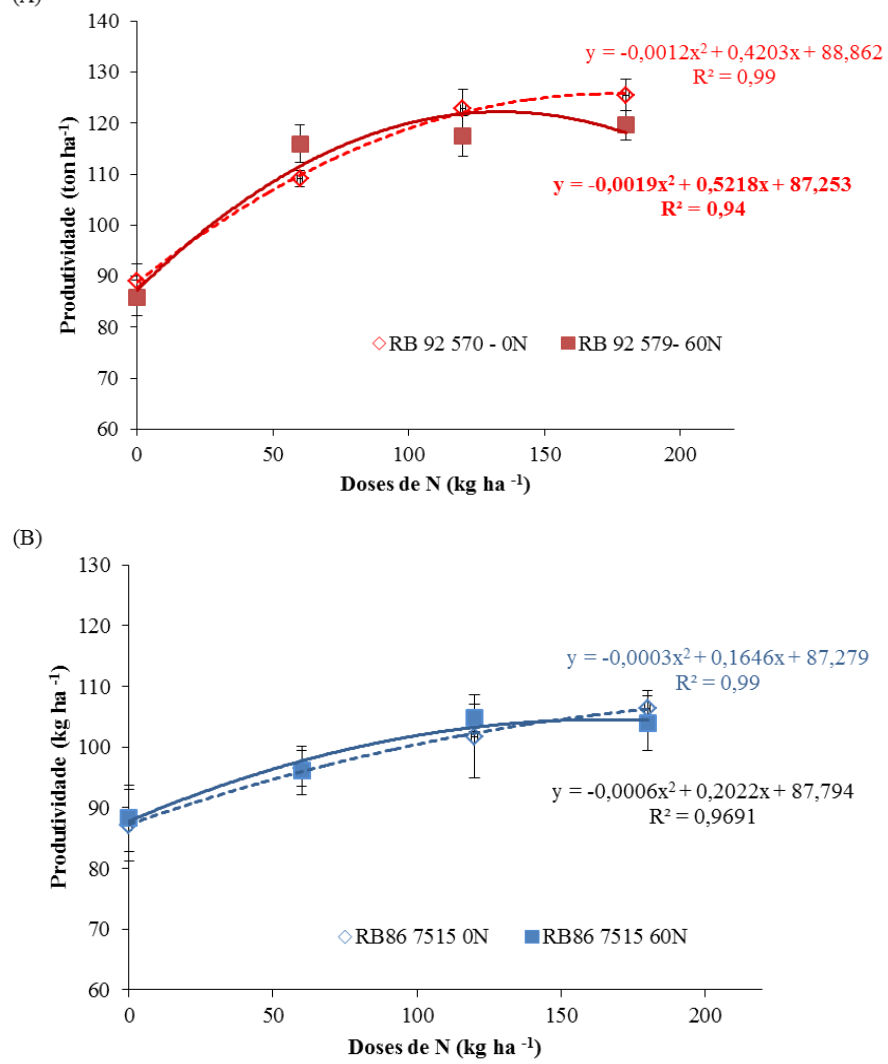

De acordo com Mello Ivo et al. (2008), a quantidade de N aplicada na adubação da cana, no Brasil, em comparação aos outros países, é uma das mais baixas, sendo de $42 \mathrm{~kg}$ de $\mathrm{N} \mathrm{ha}^{-1}$, para a cana planta, e de $80 \mathrm{~kg} \mathrm{ha}^{-1}$ para a socaria, e foi obtida para sistemas de produção que envolvem colheita de cana com o uso do fogo. Para a região Sudeste do Brasil, a recomendação de adubação fica em torno de $30 \mathrm{~kg}$ de $\mathrm{N} \mathrm{ha}^{-1}$, para a cana planta; e varia de 60 a $120 \mathrm{~kg}$ de $\mathrm{N} \mathrm{ha}^{-1}$, para socarias, a depender da produtividade esperada (RAIJ, 1996). No Nordeste, predomina o uso da dose de $60 \mathrm{~kg}$ de $\mathrm{N} \mathrm{ha}^{-1}$, com algumas usinas adicionando $80 \mathrm{~kg}$ de $\mathrm{N} \mathrm{ha}^{-1}$, para a socaria. $\mathrm{Na}$ recomendação de adubação elaborada para o Estado de Alagoas (MARINHO et al.,1980), já se destacava a necessidade de ampliação das doses de $\mathrm{N}$ em $30 \mathrm{~kg}$ de $\mathrm{N} \mathrm{ha}^{-1}$, no caso do cultivo de cana em solos muito arenosos. Isto em função dos baixos teores de matéria orgânica e, consequentemente, de nitrogênio do solo. De acordo com Vitti et al. (2008), respostas da cana-planta ao nitrogênio podem ser altas em solos de textura leve, sendo este o cenário para a maioria dos solos dos tabuleiros cultivados com cana. No presente estudo, para um Argissolo Amarelo localizado na feição geográfica dos Tabuleiros Costeiros do Nordeste, as doses de máxima eficiência econômica encontradas ficaram acima das recomendadas, variando de 126 a $124 \mathrm{~kg}$ de $\mathrm{N} \mathrm{ha}^{-1}$, para a socaria cultivada em sucessão a cana planta com $60 \mathrm{~kg}$ de N.ha ${ }^{1}$, para a RB 867515 e RB 92579, respectivamente. c) Segunda socaria

Como já descrito acima para a primeira socaria, dado que não houve efeito residual da leguminosa e do fertilizante sobre a produtividade da primeira soca, as análises na segunda socaria também se relacionaram apenas com a resposta da cana às diferentes doses de $\mathrm{N}\left(0,60,120\right.$ e $180 \mathrm{~kg}$ de $\left.\mathrm{N} \mathrm{ha}^{-1}\right)$. Polinômios de segundo grau foram ajustados para descrever as respostas da cana ao $\mathrm{N}$ ( $\mathrm{p}<0,0003$ ) (Figura 2). Assim como para a cana planta e primeira socaria, a variedade RB 92579 apresentou maiores produtividades que a RB 867515.

Figura 3. Curva de resposta de cana-de-açúcar a aplicações de nitrogênio, para segunda socaria das variedades RB 92579 e RB 867515, cultivadas em Argissolo Amarelo. Município de Coruripe, Alagoas.

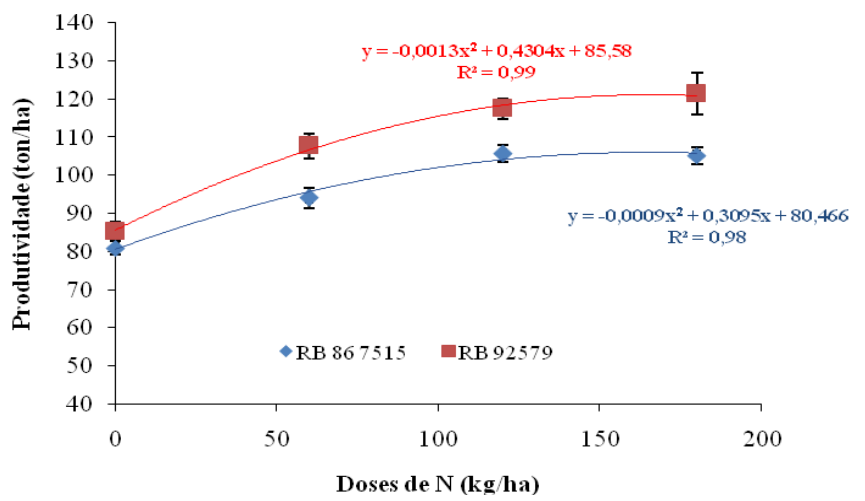

No presente estudo, para um Argissolo Amarelo, as doses de máxima eficiência econômica encontradas para a segunda socaria da cana ficaram acima das recomendadas, variando de 144 a $146 \mathrm{~kg}$ de $\mathrm{N} \mathrm{ha}^{-1}$, para a RB 867515 e RB 92579, respectivamente. Mostrando que, para estes materiais de alto rendimento, quando todos os fatores de produção estão corrigidos, doses de $\mathrm{N}$ maiores que aquelas das tabelas de recomendação existentes podem ser aplicadas. Em estudo realizado por Prado e Pancelli (2008), a resposta das duas primeiras soqueiras de cana-de-açúcar à aplicação de nitrogênio, em sistema de colheita sem queima, ocorreu apenas no segundo corte. A dose de $200 \mathrm{~kg} \mathrm{ha}^{-1}$ de $\mathrm{N}$ promoveu aumento no desenvolvimento da cultura, na nutrição da planta e na produtividade de colmos da soqueira da cana-de-açúcar. De acordo com Vitti et al. (2008), em muitos experimentos respostas lineares são relatadas até $120 \mathrm{~kg} \mathrm{ha}^{-1}$, ou as doses calculadas para as máxima produção física ultrapassam $150 \mathrm{~kg}$; porém, os aumentos obtidos a partir de $100 \mathrm{~kg} \mathrm{ha}^{-1}$ são relativamente pequenos. Esta tendência de menor resposta pode ser percebida para estas doses de $144 \mathrm{a} 146 \mathrm{~kg} \mathrm{ha}^{-1}$, uma vez que, entre as doses de 120 e $180 \mathrm{~kg}$ de $\mathrm{N} \mathrm{ha}^{-1}$ a produtividade só aumentou 2 a $3 \mathrm{t} / \mathrm{ha}$. No entanto, até a dose de $120 \mathrm{~kg} \mathrm{de} \mathrm{N} \mathrm{ha}^{-1}$ os ganhos foram expressivos para as duas variedades, sendo este de 10 e $12 \mathrm{t}$ de colmos ha ${ }^{-1}$. Tal padrão de resposta justifica a elevação da recomendação de $\mathrm{N}$ para a socaria da cana, para os Argissolos dos tabuleiros costeiros, com teores de $\mathrm{N}$ variando de $0,52 \mathrm{~g} \mathrm{~kg}^{-1}$ em superfície $(0-20 \mathrm{~cm})$, chegando a $0,41 \mathrm{~g} \mathrm{~kg}^{-1}$ aos $90 \mathrm{~cm}$ no perfil. 


\section{CONCLUSÕES}

A resposta da cana planta à rotação com crotalária e à aplicação de $60 \mathrm{~kg}$ de $\mathrm{N}^{-1}$ é expressiva, com ganhos de produtividade de colmos de $35 \mathrm{Mg} \mathrm{ha}^{-1}$ e $15 \mathrm{Mg} \mathrm{ha}^{-1}$, respectivamente; com a indicação de que a cana só responde ao $\mathrm{N}$ fertilizante na ausência da leguminosa.

$\mathrm{O}$ nitrogênio da fertilização nitrogenada em doses crescentes até $180 \mathrm{~kg} \mathrm{ha}^{-1}$ de $\mathrm{N}$ resulta em resposta quadrática na produtividade de colmos da socaria da cana, estabelecendo doses de máxima eficiência econômica variando de $124 \mathrm{~kg} \mathrm{ha}^{-1}$ a $146 \mathrm{~kg} \mathrm{ha}^{-1}$ de $\mathrm{N}$, para materiais de alto rendimento, como as variedades RB 92579 e RB 867515, cultivados em Argissolo Amarelo dos Tabuleiros Costeiros do Nordeste.

\section{AGRADECIMENTO(S)}

À Usina Coruripe, pela estrutura física e o apoio na condução dos experimentos.

\section{REFERÊNCIAS}

BARRETO, A. C.; FERNANDES, M. F. Recomendações técnicas para o uso da adubação verde em solos de Tabuleiros Costeiros. Aracaju: Embrapa Tabuleiros Costeiros, 2001. 24 p. (Embrapa Tabuleiros Costeiros. Circular técnica, 19).

BAYER, C.; AMADO, T. J. C.; TORNQUIST, C. G.; CERRI, C. E. T.; DIECKOW, J.; ZANATTA, J. A.; NICOLOSO, R. da S.; CARVALHO, P. C. de F. Estabilização do carbono no solo e mitigação das emissões de gases de efeito estufa na agricultura conservacionista. In: KLAUBERG FILHO, O.; MAFRA, A. L.; GATIBONI, L. C. (Eds). Tópico em Ciência do Solo, Viçosa, MG, 2011. Cap2., v. 7, p. 55-118.

BOLONHEZI, D.; BOLONHEZI, A. C.; CARLOS, J. A. D. Adubação verde e rotação de culturas para a cana-de-açúcar. In: LIMA FILHO, O. F.; AMBROSANO, E. J.; ROSSI, F.; CARLOS, J. A. D. (Ed.). Adubação Verde e Plantas de Cobertura no Brasil: Fundamentos e Práticas. Brasília, DF: Embrapa, 2014. v. 2. p 128-158.

FRANCO, H. C. J.; TRIVELIN, P. C. O.; FARONI, C. E.; VITTI, A. C.; OTTO, R. Stalk yield and technological attributes of planted cane as related to nitrogen fertilization. Scientia Agricola. v.67, $\mathrm{n}^{\circ} .5,2010 . \underline{10.1590 / \mathrm{S} 0103-90162010000500012}$

GAVA, G. J. C.; TRIVELIN, P. C. O.; VITTI, A. C.; OLIVEIRA, M. W. Recuperação do nitrogênio $(15 \mathrm{~N})$ da uréia e da palhada por soqueira de cana-de-açúcar (Saccharum spp.). Revista Brasileira de Ciência do Solo, v. 27, p. 621-630, 2003. $\underline{10.1590 / \mathrm{S} 0100-06832003000400006}$

KORNDÖRFER, G.; OLIVEIRA, L. A. de; KORNDÖRFER, P. H. Potassium in sugar cane cropping. Sugar Journal, v. 68, n. 5, p. 11-18, 2005.

LIMA JÚNIOR, M. A. Nitrogen nutrion of sugarcane in N.E. Brazil. 1982. 172 f. Thesis PhD University of Saskatchewan, Saskatoon, 1982.

MELLO IVO, W. M. P.; ROSSETTO, R.; SANTIAGO, A. D.; BARBOSA, G. V. S.; VASCONCELOS, J. N. Impulsionando a
Produção e a Produtividade da Cana-de- açúcar no Brasil. In: ALBUQUERQUE, A. C. S.; SILVA, A. G. (eds.). Desenvolvimento da Agricultura Tropical. Brasília: Embrapa, 2008. 1 v. cap. 8, p. $673-716$.

MOURA FILHO, G.; ALBUQUERQUE, A. W. DE; MOURA, A. B.; SANTOS, A. C. I. DOS; OLIVEIRA FILHO, M. DOS S.; SILVA, L. C. DA. Diagnose nutricional de variedades de cana-de-açúcar em argissolos. Revista Brasileira de Engenharia Agrícola e Ambiental, v. 18, p. 1102-1109, 2014. 10.1590/1807-1929/agriambi.v18n11p1102-1109

OLIVEIRA, E. C. A.; FREIRE, F. J.; OLIVEIRA, R. I.; FREIRE, M. B. G.; SIMOES NETO, D. E.; SILVA, S. A. M. Extração e exportação de nutrientes por variedades de cana-deaçúcar cultivadas sob irrigação plena. Revista Brasileira de Ciência do Solo, v. 34, n. 4, p. 1343-1352, 2010. 10.1590/S0100-06832010000400031

PRADO, R. M.; PANCELLI, M. A. Resposta de soqueiras de cana-de-açúcar à aplicação de nitrogênio em sistema de colheita sem queima. Bragantia, v. 67, n. 4, p. 951-959, 2008.

RAIJ, B. van. Avaliação da fertilidade do solo. Piracicaba: Instituto da Potassa e Fosfato, Instituto Internacional da Potassa, 1981. $142 \mathrm{p}$.

RAIJ, B. van; CANTARELlA, H.; QUAGGIO, J. A.; FURLANI, A. M. C. (Ed.). Recomendacões de adubacão e calagem para o Estado de São Paulo. 2. ed. Campinas: Instituto Agronômico, 1996. 285 p. (IAC. Boletim Tecnico, 100).

SILVA, F. C. da (Org.). Manual de análises químicas de solos, plantas e fertilizantes. Brasília, DF : Embrapa Comunicação para Transferência de Tecnologia; Rio de Janeiro: Embrapa Solos; Campinas: Embrapa Informática Agropecuária, 1999. $370 \mathrm{p}$.

SILVA, E. F. Produção e decomposição da palhada em área de cultivo de cana-de-açúcar no município de Coruripe, Alagoas .54 f. Monografia (Graduação em Agronomia), Universidade Federal de Alagoas, Rio Largo, 2013.

SILVA, P. A.; MARAFON, A. C.; MELLO IVO, W. M. P.; PAIVA, H. L.; GUIMARÃES, V. S.; NEVES, J. A. Uso de Leguminosas na Renovação de Canaviais: Espécies Recomendadas para Diferentes Épocas de Plantio nos Tabuleiros Costeiros de Alagoas. Disponível em: <https://ainfo.cnptia.embrapa.br/digital/bitstream/item/153979/ 1/cot-196.pdf.> Acesso em 20 de novembro de 2019.

VITTI, A. C.; TRIVELIN, P. C. O.; GAVA, G. J. C.; PENATTI, C. P.; BOLOGNA, I. R.; FARONI, C. E.; FRANCO, H. C. J. Produtividade da cana-de-açúcar relacionada ao nitrogênio residual da adubação e do sistema radicular. Pesquisa Agropecuária Brasileira, v. 42, n. 2, p. 249256, 2007.

VITTI, A. C.; CANTARELlA, H.; TRIVELIN, P. C. O.; ROSSETTO, R. Nitrogênio. In: DINARDO-MIRANDA, L. L.; VASCONCELOS, A. C. M.; LANDEL, M. G. A. Cana-deaçúcar. Campinas: Instituto Agronômico., 2008, pp. 239-270. 